23

\title{
Компенсация погрешностей модели центральной проекции в трехмерных сканерах
}

\author{
(C) А.А. Бенуни ${ }^{1}$, В.Я. Колючкин ${ }^{2}$ \\ ${ }^{1}$ НИИ „Радиоэлектроника и лазерная техника“ МГТУ им. Баумана, \\ 105005 Москва, Россия \\ ${ }^{2}$ Кафедра „Лазерные и оптико-электронные системы“, МГтУ им. Баумана, \\ 105005 Москва, Россия \\ e-mail: benuni_anna@mail.ru
}

Поступила в редакцию 24.05.2019 г.

В окончательной редакции 24.05.2019 г.

Принята к публикации 04.06.2019 г.

Приведены результаты исследований погрешности триангуляционных трехмерных сканеров, возникающей при использовании модели центральной проекции. Показано, что аберрации в зрачках объективов сканера могут приводить к существенным погрешностям определения координат точек поверхности объектов. Сформулировано требование к аберрационным характеристикам объективов для минимизации погрешности трехмерных сканеров и предложена модификация модели центральной проекции, позволяющая уменьшить погрешность, обусловленную аберрациями в зрачках объективов триангуляционных сканеров.

Ключевые слова: трехмерный сканер, погрешность регистрации, модель центральной проекции, дисторсия, аберрации в зрачках.

DOI: $10.21883 /$ OS.2019.10.48368.193-19

\section{Введение}

В настоящее время производится широкая номенклатура трехмерных (3D) бесконтактных оптикоэлектронных сканеров, обладающих высокими техническими характеристиками. Например, серийно выпускаемые триангуляционные 3D сканеры со структурированной подсветкой обеспечивают получение трехмерных моделей объектов с погрешностью, не превышающей $0.1 \mathrm{~mm}$ в угловом поле $\pm 20^{\circ}$ и на дальностях от 0.3 до $1.3 \mathrm{~m}$. Достижение более высоких показателей точности в пределах всей области сканирования является важной технической задачей.

Известно [1,2], что в триангуляционных сканерах погрешность оценки 3D координат точек поверхности регистрируемых объектов в значительной мере определяется погрешностями калибровки и адекватностью математической модели, которая положена в основу описания изображений, формируемых объективами каналов подсветки и регистрации изображений 3D сканера. Задаче калибровки 3D сканеров посвящен ряд работ [3-6]. Для калибровки этих приборов используют плоские тест-объекты в виде шахматной доски, а описание формирования изображений объективами каналов подсветки и регистрации изображений производят на основе модели центральной проекции с коррекцией геометрических искажений, обусловленных дисторсией. Получаемая в результате матрица калибровки включает внешние параметры, описывающие взаимное положение визирных осей каналов 3D сканера, а также внутренние параметры, в том числе фокусные расстояния и коэффициенты аппроксимации дисторсии объективов.
Достоинством модели центральной проекции является относительная простота алгоритмов обработки регистрируемых изображений. Но эта модель не учитывает того факта, что из-за аберраций в зрачках реальных объективов может изменяться положение входного и выходного зрачков для различных значений углового поля $[7,8]$. Вследствие этого возникают погрешности калибровки 3D сканеров, которые приводят к погрешностям определения координат точек поверхностей регистрируемых объектов.

Целью исследований, изложенных в настоящей работе, является повышение точности триангуляционных 3D сканеров со структурированной подсветкой. Для достижения этой цели проведен анализ условий применимости модели центральной проекции и предложена модификация модели центральной проекции, позволяющая уменьшить влияние аберраций в зрачках объективов триангуляционного сканера на погрешности регистрации $3 \mathrm{D}$ образов объектов.

\section{Оценка погрешностей при использовании модели центральной проекции}

Чтобы оценить погрешности, возникающие при использовании модели центральной проекции, рассмотрим ход лучей в объективе канала регистрации изображений 3D сканера при наличии сферической аберрации в зрачках. Отметим, что все последующие рассуждения справедливы и для объектива канала подсветки триангуляционного сканера, но в обратном ходе лучей. 


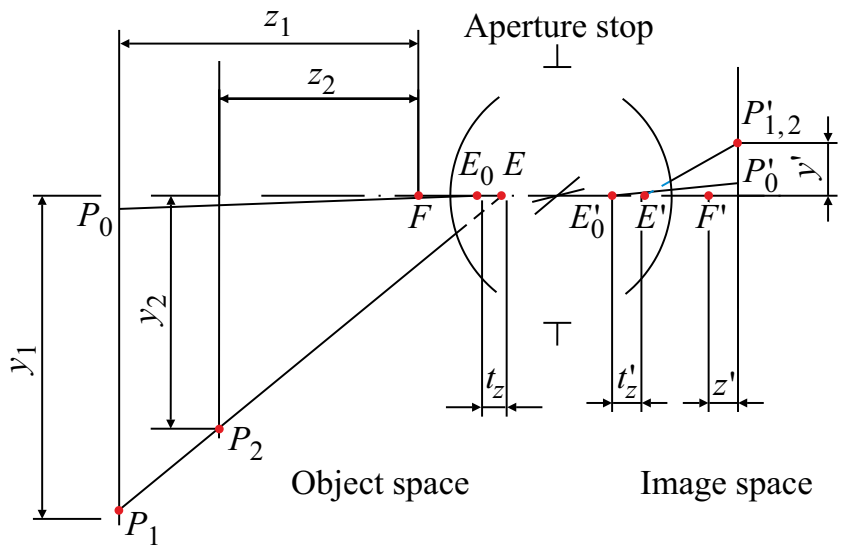

Рис. 1. Ход параксиального $P_{0} E_{0}$ и реальных $P_{1} E$ и $P_{2} E$ главных лучей в оптической системе при наличии сферической аберрации в зрачках объектива.

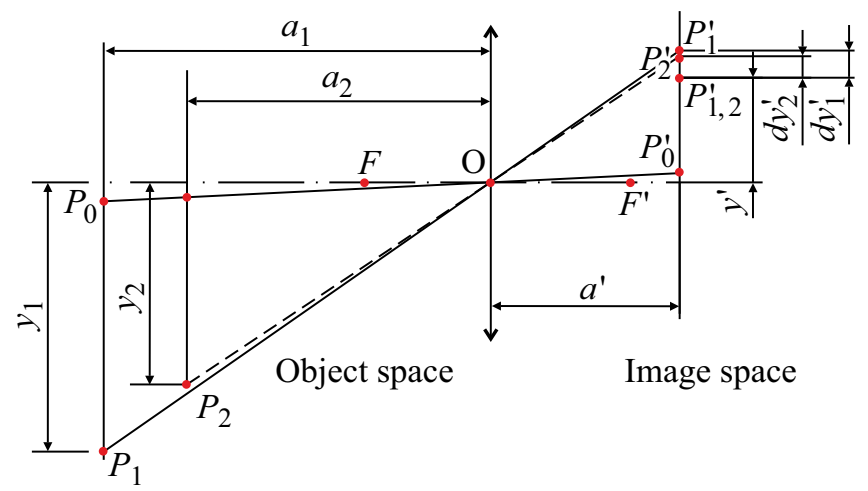

Рис. 2. Ход главных лучей в модели центральной проекции.

На рис. 1 показан ход параксиального луча $P_{0} E_{0}$ и реальных главных лучей $P_{1} E$ и $P_{2} E$ в объективе с некомпенсированной сферической аберрацией в зрачках. Точки $P_{1}$ и $P_{2}$, расположенные в пространстве предметов на расстояниях $z_{1}$ и $z_{2}$, лежат на одной линии, совпадающей с главным лучом $P_{1,2} E$. Главные лучи $E^{\prime} P_{1}^{\prime}$ и $E^{\prime} P_{2}^{\prime}$ в пространстве изображений совпадают и пересекают плоскость изображения в точке $P_{1,2}^{\prime}$. В точке $E_{0}$ находится центр входного зрачка в параксиальном приближении, а в точке $E$ - центр входного зрачка для главных лучей $P_{1} E$ и $P_{2} E$. Продольная сферическая аберрация во входном и выходном зрачках соответственно оценивается отрезками $t_{z}$ и $t_{z}^{\prime}$.

На рис. 2 приведен ход лучей для модели центральной проекции объектива канала регистрации. Согласно этой модели, все лучи проходят через одну точку $O$, определенную как центр проекции. При таком допущении главные лучи $\mathrm{P}_{1} O$ и $\mathrm{P}_{2} O$ для точек $P_{1}$ и $P_{2}$, выбранных на рис. 1, не совпадут и пересекут плоскость изображения в точках $P_{1}^{\prime}$ и $P_{2}^{\prime}$. Из-за наличия геометрических искажений, обусловленных дисторсией, изображение точек $P_{1}$ и $P_{2}$ находится в точке $P_{1,2}^{\prime}$ и отстоит от идеальных точек центральных проекций на различные величины $d y_{1}^{\prime}$ и $d y_{2}^{\prime}$. Следовательно, допущение, принятое в модели центральной проекции, о том, что дисторсия зависит только от направления главного луча, не справедливо при наличии сферической аберрации в зрачках.

Для оценки значений погрешности модели центральной проекции, возникающей вследствие наличия сферической аберрации в зрачках объективов, был проведен численный анализ зависимости дисторсии от дальности до объекта на примере нескольких объективов. На рис. 3 приведены оптические схемы объективов, выбранных из каталога объективов Zemax Zebase, в том числе проекционных объективов L011 и L021, F012 и объективов V002, V008 с телецентрическим ходом лучей в пространстве изображений. Анализ проводился путем расчета хода лучей в программе Zemax. При
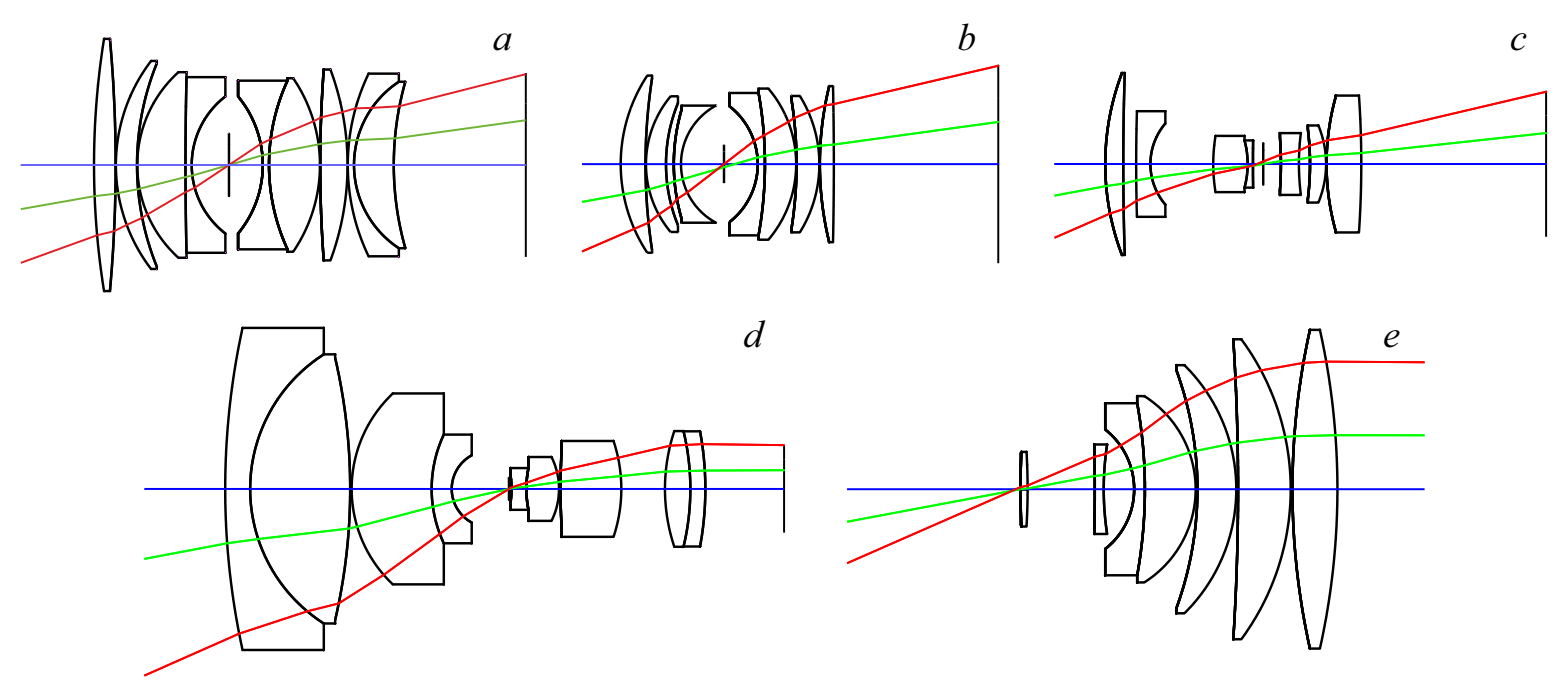

Рис. 3. Оптические схемы объективов L021 (a), L011 (b), F012 (c), V002 (d), V008 (e). 

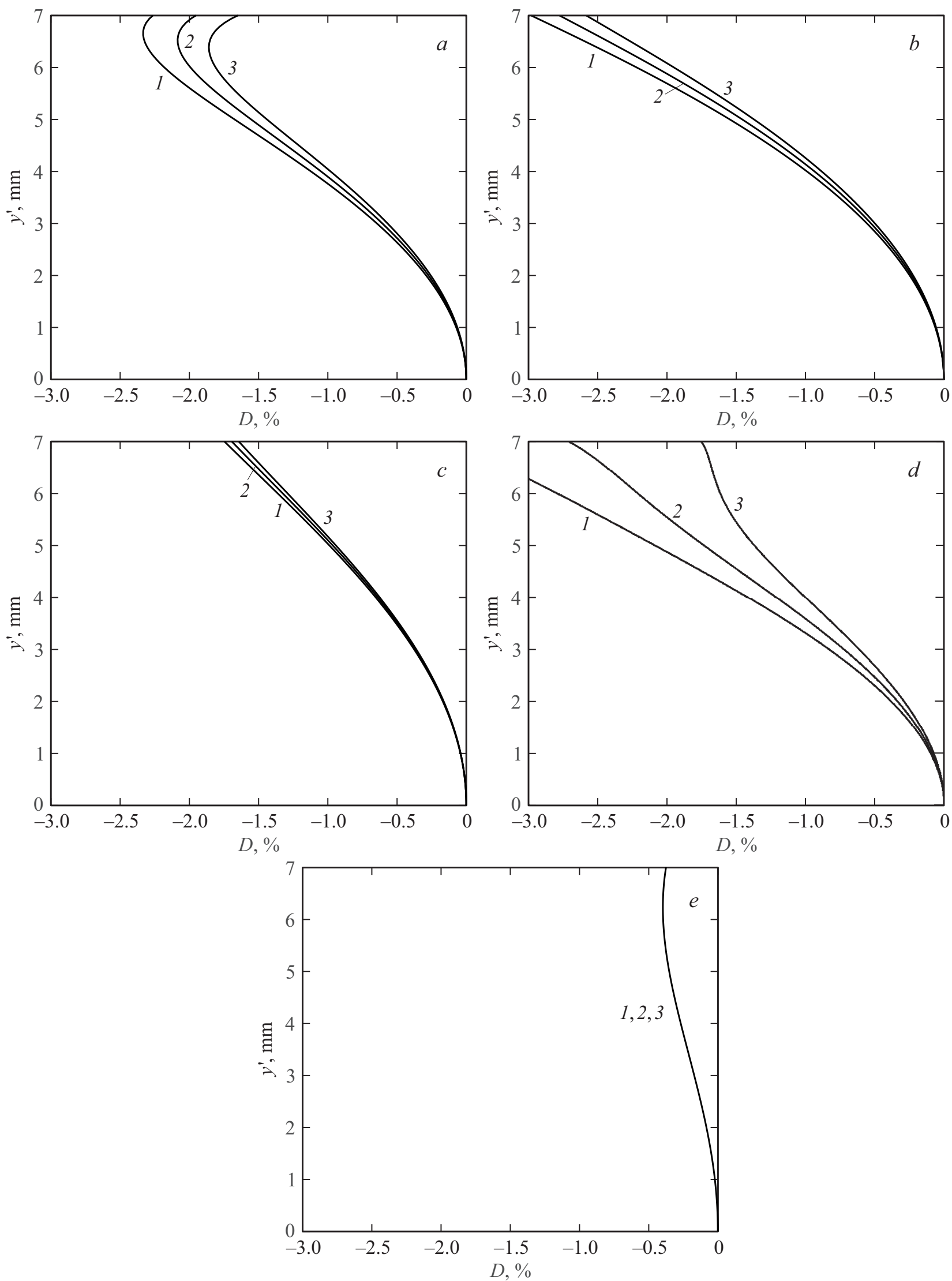

Рис. 4. Графики зависимости дисторсии $D, \%$ от координаты $y^{\prime}$ в плоскости изображения при дальностях до объекта $z=300(1)$, 500 (2) и $1300 \mathrm{~mm}(3)$ для объективов L021 (a), L011 (b), F012 (c), V $002(d), \mathrm{V} 008(e)$. 

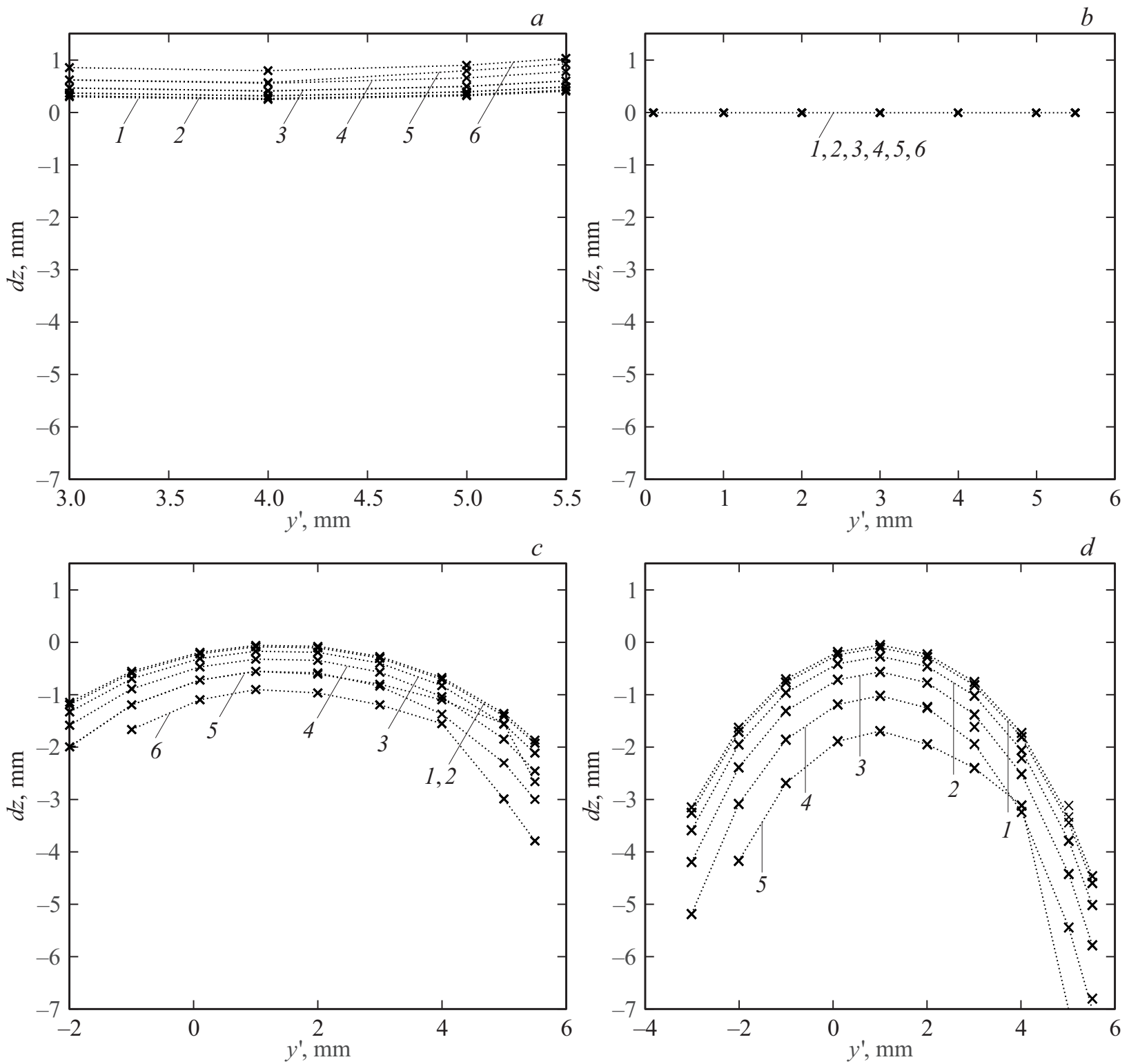

Рис. 5. Распределения погрешности измерения координаты $d z\left(y^{\prime}\right)$ при использовании модели центральной проекции для объективов L021 в приемной и передающей системе на дальностях до объектов $z=300(a), 500(b), 700(c)$ и 1300 mm $(d)$ для сечений $x^{\prime}=0(1) ; \pm 1(2) ; \pm 2(3) ; \pm 3(4) ; \pm 4(5) ; \pm 5 \mathrm{~mm}$ (6).

этом объективы были отмасштабированы и приведены к единому фокусному расстоянию, равному $16 \mathrm{~mm}$. Плоскость изображения выбиралась, как плоскость наилучшей установки для дальности $500 \mathrm{~mm}$ до объекта в пространстве предметов. Угловое поле составляло $\pm 20^{\circ}$, а расстояния до объекта сканирования варьировались в пределах от 300 до $1300 \mathrm{~mm}$. Полученные в результате расчетов зависимости дисторсии от координаты $y^{\prime}$ главного луча в плоскости изображений для значений дальности до объекта $z=300,500$ и 1300 mm приведены на рис. 4.

Из анализа полученных графиков аберраций следует, что только для объектива V008, дисторсия не зависит от дальности до объекта, так как в этом объективе апер- турная диафрагма совпадает с оправой первой линзы, и сферическая аберрация во входном зрачке отсутствует. Для объектива L021 в указанном диапазоне дальностей до объекта максимальная разность значений дисторсии составляет около $0.5 \%$ при угловом поле $\pm 20^{\circ}$, а для объектива V002 - около 1\%. Зависимость дисторсии от дальности для объективов L011, F12 - менее выражена.

Для оценки погрешностей 3D сканера был проведен расчет дальности до объекта при использовании модели центральной проекции. Расчет выполнялся при условии, что оптические оси каналов подсветки и регистрации изображений 3D сканера параллельны, а расстояние между осями равно $150 \mathrm{~mm}$. В качестве тест-объектов 

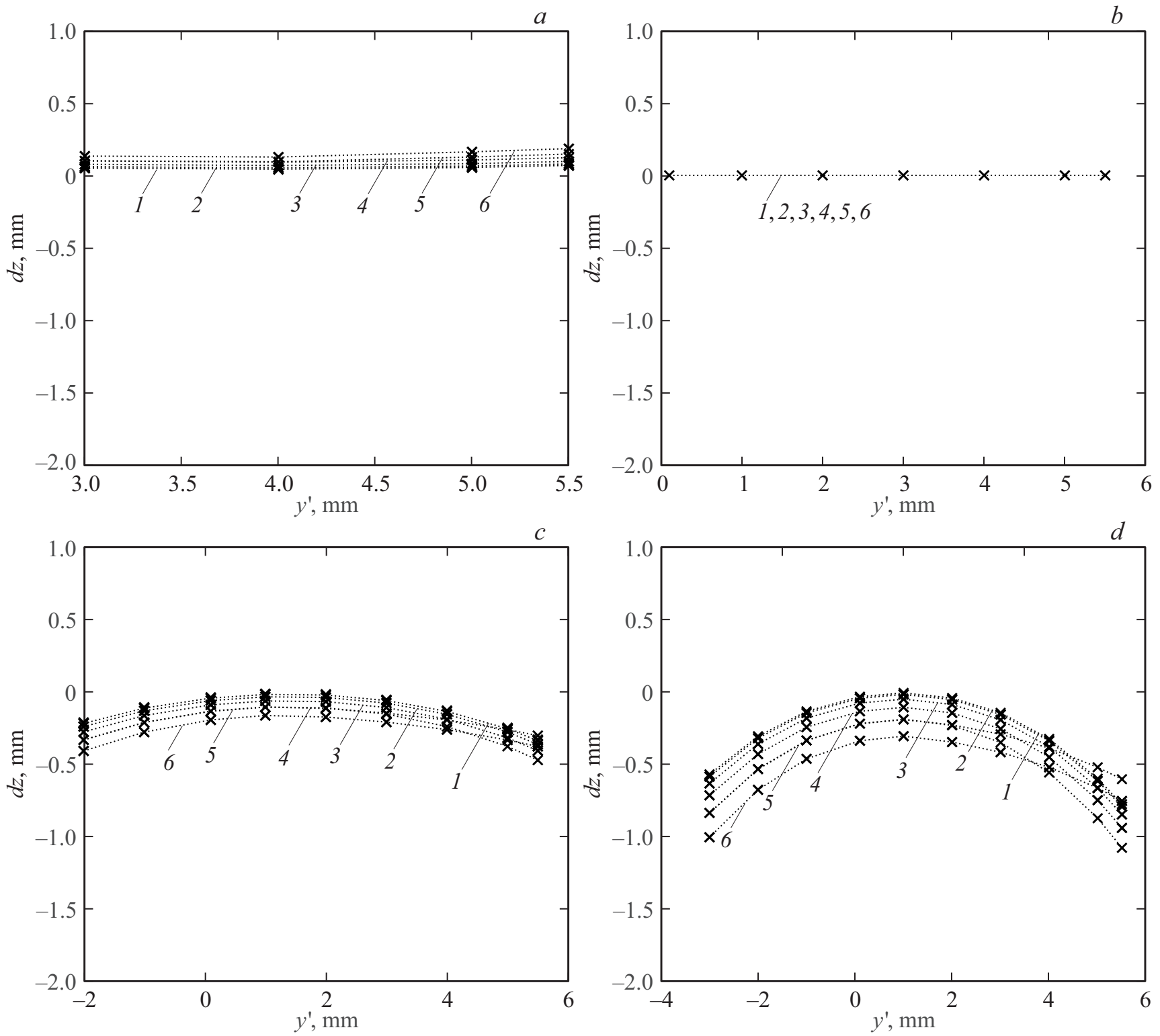

Рис. 6. Распределения погрешности измерения координаты $d z\left(y^{\prime}\right)$ при использовании модели центральной проекции для объективов F012 в приемной и передающей системе на дальностях до объектов $z=300(a), 500(b), 700(c)$ и 1300 mm $(d)$ для сечений $x^{\prime}=0(1) ; \pm 1(2) ; \pm 2(3) ; \pm 3(4) ; \pm 4(5) ; \pm 5 \mathrm{~mm}(6)$.

использовались плоскости, расположенные перпендикулярно оптическим осям на фиксированных расстояниях $z$. В процессе вычислений координат тест-объекта производилась компенсация дисторсии для дальности $z^{*}=500 \mathrm{~mm}$.

На рис. 5 и 6 приведены графики зависимости погрешности $d z\left(y^{\prime}\right)$ измерения координаты $z$ от координаты $y^{\prime}$ на сенсоре при использовании модели центральной проекции для нескольких значений дальности $z$ до объекта и для различных сечений по оси $x^{\prime}$, соответствующих значениям $x^{\prime}=0 ; \pm 1 ; \pm 2 ; \pm 3 ; \pm 4 ; \pm 5 \mathrm{~mm}$.

Как следует из приведенных графиков, при удалении от плоскости коррекции дисторсии, соответствующей $z^{*}=500 \mathrm{~mm}$, полученная 3D модель тестовой плоской поверхности „изгибается“. В частности, при использовании в каналах подсветки и регистрации 3D сканера объективов модели L021 погрешность определения дальности для $z=1300 \mathrm{~mm}$ на краю поля превышает значение $d z=5 \mathrm{~mm}$.

Из проведенного анализа можно сделать вывод, что применение модели центральной проекции без учета зависимости дисторсии от дальности для некоторых типов объективов может приводить к существенным погрешностям определения координат точек поверхности объектов. Для минимизации этих погрешностей к объективам каналов подсветки и регистрации изображений 3D сканеров следует либо предъявлять жесткие требования к сферической аберрации в зрачках, либо 


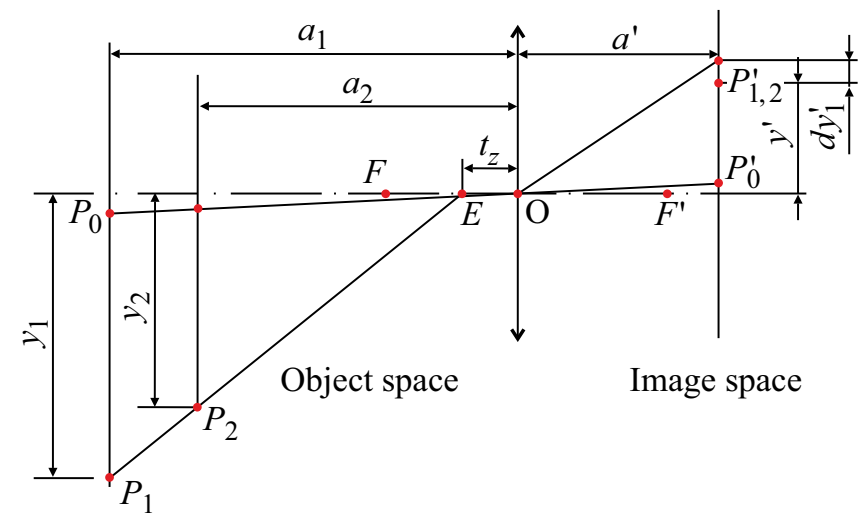

Рис. 7. Ход главных лучей в модифицированной модели центральной проекции объектива.
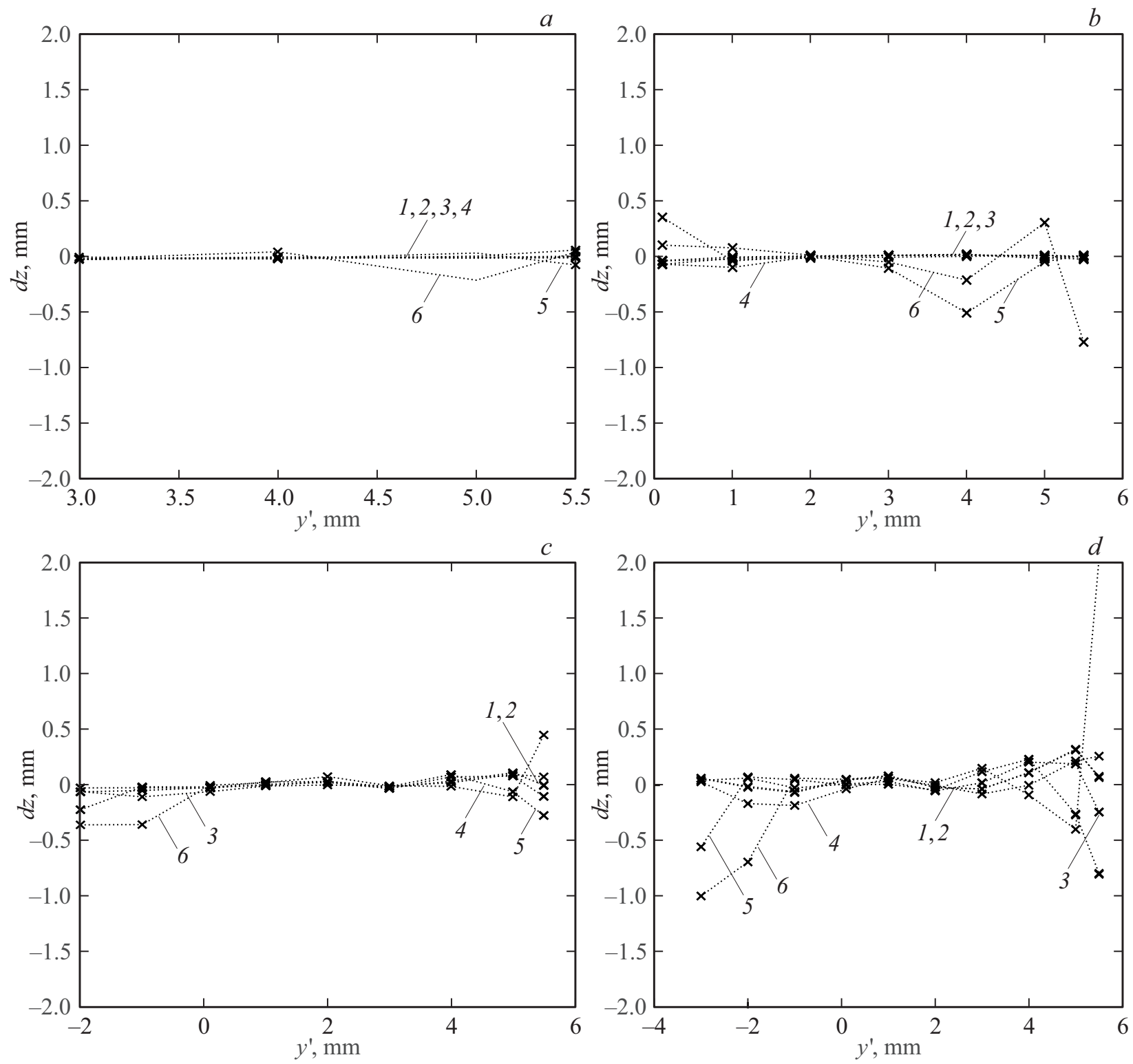

Рис. 8. Значения погрешности измерения координаты $d z\left(y^{\prime}\right)$ при использовании модифицированной модели центральной проекции на дальностях до объектов $z=300(a), 500(b), 700(c)$ и $1300 \mathrm{~mm}(d)$ для сечений $x^{\prime}=0(1) ; \pm 1(2) ; \pm 2(3) ; \pm 3(4) ; \pm 4(5)$; $\pm 5 \mathrm{~mm}(6)$. 
учитывать зависимость дисторсии от дальности в модели центральной проекции.

\section{Модифицированная модель центральной проекции}

Известен метод учета зависимости дисторсии от дальности в модели центральной проекции объективов $3 \mathrm{D}$ сканеров с некомпенсированной сферической аберрацией в зрачках, который заключается в следующем [9]: рассчитываются зависимости дисторсии от поперечных координат точек объектов для некоторых двух фиксированных плоскостей пространства предметов, производится предварительная оценка дальности до точек поверхности сканируемого объекта и по формулам, выведенным авторами работы [7,8], выполняется расчет дисторсии объективов каналов подсветки и регистрации для этого значения дальности. Полученные значения дисторсии учитываются при определении 3D координат точек поверхности объекта. Описанный метод увеличивает временные затраты, так, определение координат производится за два этапа вычислений.

В связи с этим предложена модифицированная математическая модель центральной проекции объективов канала подсветки и регистрации изображений, которая иллюстрируется рис. 7. В соответствии с этой моделью центр проекции $E$ в пространстве предметов смещен относительно параксиального положения входного зрачка на величину $t_{z}$, которая определяет продольную сферическую аберрацию во входном зрачке объектива.

Для осесимметричного объектива смещение $t_{z}$ зависит от расстояния $r^{\prime}$ от точки $P_{1,2}^{\prime}$ до оптической оси, т.е. $t_{z}\left(r^{\prime}\right)$, причем $r^{\prime}=\sqrt{x^{\prime 2}+y^{\prime 2}}$.

Тогда координаты точки в пространстве предметов (рис. 7) можно определить по формулам

$$
\begin{aligned}
& x=\left(a-t_{z}\left(r^{\prime}\right)\right) \frac{x^{\prime}}{a^{\prime}} D\left(r^{\prime}\right), \\
& y=\left(a-t_{z}\left(r^{\prime}\right)\right) \frac{y^{\prime}}{a^{\prime}} D\left(r^{\prime}\right),
\end{aligned}
$$

где $t_{z}\left(r^{\prime}\right)$ - продольная сферическая аберрация во входном зрачке; $D\left(r^{\prime}\right)$ - функция, описывающая радиальную дисторсию, которую обычно аппроксимируют степенным рядом

$$
D\left(r^{\prime}\right)=\left(1+k_{1} r^{\prime 2}+k_{2} r^{\prime 4}+k_{3} r^{\prime 6}\right)
$$

Для аппроксимации зависимости продольной сферической аберрации от расстояния $r^{\prime}$ предлагается использовать степенной ряд, имеющий вид

$$
t_{z}\left(r^{\prime}\right)=t_{1} r^{\prime 2}+t_{2} r^{\prime 4}+t_{3} r^{\prime 6} .
$$

Предложенная модель была апробирована для объектива L021. В результате расчета было определено расстояние $a^{\prime}=16.4238 \mathrm{~mm}$ от точки $O$ - центра проекции в пространстве изображений до плоскости изображения. В результате аппроксимации были получены следующие значения коэффициентов, входящих в формулы (3) и (4): $t_{1}=-0.0367 ; t_{2}=5.667 \cdot 10^{-5} ; t_{3}=-1.21 \cdot 10^{-5}$; $k_{1}=-4.29 \cdot 10^{-5} ; \quad k_{2}=5.72 \cdot 10^{-7} ; \quad k_{3}=-1.92 \cdot 10^{-8}$. При этом относительная погрешность аппроксимации зависимостей $D\left(r^{\prime}\right)$ и $t_{z}\left(r^{\prime}\right)$ не превысила $0.03 \%$.

Отметим, что при расчетах координаты в пространстве изображений определялись как точки пересечения плоскости изображения с главными лучами.

Для оценки эффективности предложенной модели были проведены численные эксперименты по оценке погрешности $d z\left(y^{\prime}\right)$ определения координат поверхности объекта 3D сканером, в котором в каналах подсветки и регистрации изображений используется объектив типа L021. B качестве тест-объектов, как и в проведенном выше анализе, использовались плоскости, расположенные перпендикулярно оптическим осям на фиксированных расстояниях $z$. Расчетные значения трехмерных координат точек определялись из условия пересечения лучей каналов подсветки и регистрации, которые описывались в соответствии с формулами (1)-(4).

На рис. 8 представлены распределения погрешности измерения координаты $d z\left(y^{\prime}\right)$ на дальностях $z=300$, 500, 900 и $1300 \mathrm{~mm}$. Из сравнения графиков, представленных на рис. 5 и 8, следует, что погрешность оценки формы 3D модели плоского тест-объекта, полученной с использованием модифицированной модели для различных дальностей до объектов, примерно в 10 раз меньше, чем при использовании модели центральной проекции.

\section{Заключение}

На основании полученных результатов можно сделать вывод о том, что предложенная математическая модель позволяет существенно уменьшить СКО погрешности определения формы 3D сканерами, предназначенными для регистрации объектов в широком диапазоне дальностей. Данная модель может быть использована в алгоритмах программного обеспечения как 3D сканеров со структурированной подсветкой, так и стереоскопических 3D сканеров.

\section{Список литературы}

[1] Trobina M. Error Model of a Coded-Light Range Sensor: Technical Report: BIWI-TR-164 / ETH-Zentrum. Zurich, 1995. $36 \mathrm{p}$.

[2] Volodine Y. Statistical Error Model of Active Triangulation Method for CAI [Электронный pecypc] GraphiCon 2003 Proceedings. URL: http://graphicon.ru/ html/2003/Proceedings/Technical/ Volodine.pdf (дата обращения 20.09.2016)

[3] Zhang Z. A Flexible New Technique for Camera Calibration Technical Report MSR-TR-98-71 Microsoft Research Microsoft Corporation One Microsoft Way Redmond, WA 98052. 
[4] Camera Calibration and 3-D Vision [Электронный peсурс] Режим доступа: URL https://www.mathworks.com/ help/vision/camera-calibration-and-3-d-vision.html

[5] Володин Ю.С., Орлов А.В., Михайлов Б.Б. // Труды Междунар. конф. с элементами науч. Школы для молодежи. СПб.: Политехника-сервис, 2010. С. 314-322.

[6] Moreno D. Simple, Accurate and Robust Projectorcamera Calibration. [Электронный ресурс] Режим доступа: URL http://mesh.brown.edu/calibration/files/Projector\%20 Calibration20Presentation.pdf

[7] Русинов М.М. Техническая оптика: Учеб. пособие для вузов. Л.: Машиностроение, 1979. 488 с.

[8] Magill A. // J. Research of the National Bureau of Standards. 1955. V. 54. N 3.

[9] Bräuer-Burchardt C., Heinze M., Munkelt C., Kühmstedt P., Notni G. Distance Dependent Lens Distortion Variation in 3D Measuring Systems Using Fringe Projection [Электронный ресурс] Режим доступа: http://www.macs.hw.ac.uk/ bmvc2006/papers/150.pdf 